\title{
ÓBITO MATERNO DEVIDO À SRAG CAUSADA POR COVID-19: UM RELATO DE CASO
}

\author{
Maternal death due to SRAG caused by COVID-19: a case report
}

\author{
Juliana F. Soares', Fernanda G.O. Nogueira', Daniel J.M. Medeiros Lima². \\ 1 Acadêmica, Faculdade de Medicina de Campos, Campos dos Goytacazes - Rio de Janeiro, Brasil. \\ 2 Professor no Departamento de Fisiologia e Farmacologia, Faculdade de Medicina de Campos, Campos dos \\ Goytacazes - Rio de Janeiro, Brasil.
}

Hospital dos Plantadores de Cana

Endereço: Av. José Alves de Azevedo, 337 - Pq. Rosario - Campos dos Goytacazes

Juliana Fraga Soares

Endereço: R. Epitácio Pessoa, 159, apto 103 - Pq. Leopoldina - Campos dos Goytacazes/RJ

Telefone: (22) 997297948 - E-mail de correspondência: julianafrsoares@gmail.com

\section{Resumo}

Existem poucos casos de gestantes com COVID-19 descritos na literatura, sendo a maioria descrito como formas brandas da doença. As evidências e informações acerca da infecção in útero e teste neonatal positivo precoce são limitados. Paciente gestante, 26 anos, sem comorbidades, deu entrada na unidade com quadro de mal-estar, febre, tosse e dispneia progressiva há três dias. Foi transferida para Unidade de Terapia Intensiva (UTI) após evolução para insuficiência respiratória, sendo necessário ventilação mecânica no décimo dia do início da doença. A paciente foi submetida a parto cesárea e o isolamento neonatal foi realizado imediatamente após o nascimento, sem retardo do pinçamento do cordão umbilical ou contato pele a pele entre mãe e bebê. Relatamos uma apresentação grave de COVID-19 durante a gravidez, com posterior evolução pata óbito materno devido a complicações da doença.

Palavras-chave: gestação, Síndrome Respiratória Aguda Grave, COVID-19, coronavírus

\begin{abstract}
There are few cases of pregnant women with COVID19 described in the literature, most of which are described as mild forms of the disease. Evidence and information about infection in utero and early positive neonatal testing are limited. Pregnant patient, 26 years old, without comorbidities, was admitted to the unit with malaise, fever, cough and progressive dyspnea for three days. She was transferred to the Intensive Care Unit (ICU) after evolution to respiratory failure, requiring mechanical ventilation on the tenth day of the disease onset. The patient underwent a cesarean delivery and neonatal isolation was performed immediately after birth without delayed umbilical cord clamping or skin to skin contact between mother and baby. We report a severe presentation of COVID-19 during pregnancy, with subsequent evolution to maternal death due to complications of the disease.
\end{abstract}

Keywords: pregnancy, Severe Acute Respiratory Syndrome, COVID-19, coronavirus 


\section{Introdução}

O novo coronavírus, denominado SARSCoV-2, foi identificado na cidade de Wuhan, na China, após um surto de pneumonia de origem desconhecida em dezembro de 2019. O vírus pertence ao gênero $\beta$-coronavirus e à família Coronaviridae e seu genoma é RNA de fita simples de sentido positivo (+ ssRNA). A doença foi intitulada COVID-19 (coronavirus disease 2019) e declarada como epidemia em 11 de março de $2020^{1},{ }^{2}$. Seu espectro clínico varia de infecções assintomáticas a pneumonias graves e apresenta como principal complicação a Síndrome Respiratória Aguda Grave(SRAG), sendo esta a principal causa de morte ${ }^{1}$.

Devido ao conhecimento adquirido nas epidemias de outros coronavírus, como SARS-CoV e MERS-CoV, mulheres grávidas são sabidamente mais susceptíveis a infecções respiratórias graves em decorrência das alterações fisiológicas da gestação, dessa forma, as consequências da COVID19 em gestantes tornaram-se uma preocupação ${ }^{3},{ }^{4}$.

\section{Objetivo}

Relatar o caso de uma paciente gestante com COVID-19, cuja evolução da doença culminou com parto cesárea de emergência e posterior óbito materno em decorrência da evolução do quadro para Síndrome Respiratória Aguda Grave (SRAG), sem maiores comprometimentos para o bebê, correlacionando as alterações fisiológicas da gestação que propiciam maior agravamento da doença.

\section{Relato de caso}

Paciente do sexo feminino, 26 anos, grávida, apresentava história de aproximadamente dez dias de sintomas gripais e três dias de dispneia. Procurou atendimento na emergência com quadro de febre, tosse e dispneia progressiva e, devido a evolução para insuficiência respiratória, foi transferida para a Unidade de Terapia Intensiva (UTI) às 31 semanas de gestação.

Ao exame físico na admissão, a paciente estava taquicárdica, taquidispneica, saturação de oxigênio $65 \%$, com murmúrio vesicular globalmente abolido e com cianose de extremidade. A paciente foi intubada e submetida à ventilação mecânica devido ao quadro de insuficiência respiratória severa, porém, imediatamente após a intubação orotraqueal, evoluiu com parada cardiorrespiratória
(PCR) com atividade elétrica sem pulso (AESP) seguida de assistolia. Foram realizadas as manobras de reanimação cardiopulmonar (RCP) e obteve-se o retorno do ritmo sinusal após 7 minutos.

A sedação da paciente foi feita com Midazolan e Fentanil e bloqueio neuromuscular com Rocurônio. Foi iniciada a administração de Ceftriaxona, Claritromicina, Metilprednisolona e Enoxaparina. Os exames laboratoriais mostraram hiperglicemia, hipercreatinemia, hiperuricemia, elevação das transaminases hepáticas e bilirrubinas, hipoalbuminemia, hipocalcemia, discreto encurtamento do TTPa e acidose mista na gasometria arterial (Tabela 1).

Após avaliação obstétrica, constatou-se que feto estava estável e através de ultrassonografia foi observada oligodramnia acentuada. Devido ao risco de vida materno-fetal e considerando a possibilidade de melhora da resposta inflamatória da mãe após a interrupção da gestação, a cesárea foi realizada na UTI. O neonato pesava $1330 \mathrm{~g}$ e sua pontuação na escala de Apgar foi 1 , tanto no primeiro quanto no quinto minuto. $O$ recém-nato nasceu em apneia, hipotônico e bradicárdico, sendo necessária intubação e massagem cardíaca com administração de Epinefrina. Obteve melhora da frequência cardíaca e foi encaminhado para a UTI neonatal, onde foi extubado após melhora clínica e permaneceu internado para ganho de peso.

A cesárea não teve intercorrências, mas o sangramento operatório foi moderado, provavelmente devido ao uso de anticoagulação plena. Não houve retardo do pinçamento do cordão umbilical ou contato pele a pele entre mãe e bebê. Foram descartadas complicações cirúrgicas inicialmente. A paciente evoluiu com SRAG e insuficiência renal aguda, chegando a apresentar clearence de creatinina de $10 \mathrm{ml} / \mathrm{min} / 1.73 \mathrm{~m} 2$, portanto, sendo necessário tratamento dialítico. Após 13 dias de internação, a paciente evoluiu com outra PCR em AESP seguida de assistolia devido à acidose respiratória, retornando ao ritmo sinusal após 15 minutos de RCP. Foi adotada a posição de prona, obtendo-se melhora dos parâmetros gasométricos. Cinco dias depois a paciente foi à óbito em decorrência de SRAG.

\section{Discussão}

Relatamos uma apresentação clínica grave de COVID-19 durante a gravidez, levando ao parto prematuro, insuficiência respiratória, ventilação 
mecânica e posterior óbito materno. O curso da doença comumente descrito na gravidez é o de uma infecção assintomática ou pneumonia leve, e a maioria dos casos são solucionados sem a necessidade de adiantamento do parto ${ }^{4}, 5,6$. Todavia, sabe-se que a gestação resulta em mudanças fisiológicas únicas e significativas, principalmente relacionadas com o sistema imunológico e respiratório, o que teoricamente tornam as mulheres grávidas mais suscetíveis a infecções virais ${ }^{7}$.

No campo da imunologia existem três estágios: no primeiro trimestre, há uma cadeia próinflamatória que garante que a invasão trofoblástica ocorra sem que haja o reconhecimento do antígeno paterno como um corpo estranho; no segundo trimestre (13 a 27 semanas), uma resposta antiinflamatória é extremamente necessária, a fim de que o crescimento fetal adequado ocorra ao mesmo tempo em que se previna o início espontâneo do trabalho de parto; no terceiro trimestre, o estímulo volta para um estado pró-inflamatório, com a finalidade de que se concretize o parto, a expulsão do bebê. Essas três etapas são marcadas por um importante equilíbrio, que quando quebrado por infecções virais, como no caso da COVID-19, pode ocasionar sérias complicações tanto maternas quanto fetais ${ }^{8}$.

Em tese, durante o estágio próinflamatório, pacientes grávidas seriam mais suscetíveis a desenvolver o que chamamos de tempestade de citocinas, resultado de uma super produção de citocinas e quimiocinas, sendo um importante indicador de gravidade na infecção por SARS-CoV- $2^{9}$.

Da mesma forma, a fisiologia pulmonar durante a gravidez também sofre alterações hormonais e funcionais que tornam as gestantes menos tolerantes à hipóxia. Isso ocorre devido ao fato de que, desde o início da gestação, os níveis elevados de progesterona atuam de forma direta no tronco encefálico, fazendo com que ocorra um aumento da frequência respiratória e o volume corrente, ao mesmo tempo em que a complacência da parede torácica diminui, assim como a resistência das vias aéreas ${ }^{10}$. Além disso, no último trimestre da gestação, o útero restringe o diafragma, promovendo uma diminuição da capacidade pulmonar total ${ }^{11}$. A hipoxemia que surge de uma infecção pulmonar pode levar à vasoconstrição e restrição de crescimento intrauterino (RCIU) ${ }^{12}$.

Essas adaptações respiratórias em associação com as alterações imunológicas colocam as gestantes em risco de desenvolver infecções respiratórias mais graves ${ }^{13}$, uma vez que estes sistemas são sítios de atuação do SARS-CoV-2.

Em relatos anteriores, a maioria dos partos foi realizado por cesárea, assim como no caso descrito. No caso da paciente, a cesariana pré-termo foi realizada devido à gravidade do quadro clínico, visto que ela precisou ser submetida à IOT, apresentava SRAG e teve uma PCR. Embora existisse uma oligodramnia, o bebê não estava em sofrimento fetal, portanto, a decisão de adiantar o parto foi somente para prevenir possíveis complicações futuras para a mãe ou para a criança, sendo inicialmente descartadas possíveis complicações do quadro devido à cirurgia. Ainda não há evidências expressivas de que a interrupção da gestação seja protetora e melhore a resposta inflamatória. Além disso, como também não existem evidências de transmissão vertical, o parto vaginal não é contraindicado, dessa forma, o tipo de parto é escolhido de acordo com o quadro clínico e obstétrico, sendo a cesárea mais utilizada em casos graves que requerem partos emergentes ${ }^{4},{ }^{13}$.

\section{Conclusão}

O caso descrito evidencia a vulnerabilidade de gestantes às infecções respiratórias e ressalta a importância deste grupo ser considerado de risco para o agravamento da COVID-19. Contudo, os dados a respeito da infecção por SARS-CoV-2 em grávidas ainda são escassos, portanto, tornam-se essenciais estudos acerca da manifestação da doença e complicações materno-fetais. Além disso, maiores investigações sobre a existência de benefícios da interrupção da gestação e de transmissão vertical são imprescindíveis. 


\section{Referências Bibliográficas}

1. Ministério da Saúde (BR). Protocolo de manejo clínico da Covid-19 na Atenção Especializada. 2020.

2. Chen Y, Liu Q, Guo D. Emerging coronaviruses: genome structure, replication, and pathogenesis. Journal of medical virology. 2020 Apr;92(4):418-23.

3. Wastnedge EA, Reynolds RM, van Boeckel SR, Stock SJ, Denison FC, Maybin JA, Critchley HO. Pregnancy and COVID-19. Physiological reviews. 2021 Jan 1;101(1):303-18.

4. Dashraath P, Wong JL, Lim MX, Lim LM, Li S, Biswas A, Choolani M, Mattar C, Su LL. Coronavirus disease 2019 (COVID-19) pandemic and pregnancy. American journal of obstetrics and gynecology. 2020 Jun 1;222(6):521-31.

5. Liu D, Li L, Wu X, Zheng D, Wang J, Yang L, Zheng C. Pregnancy and perinatal outcomes of women with coronavirus disease (COVID-19) pneumonia: a preliminary analysis. American journal of roentgenology. 2020 Jul;215(1):12732.

6. Chen H, Guo J, Wang C, Luo F, Yu X, Zhang W, Li J, Zhao D, Xu D, Gong Q, Liao J. Clinical characteristics and intrauterine vertical transmission potential of COVID-19 infection in nine pregnant women: a retrospective review of medical records. The lancet. 2020 Mar 7;395(10226):809-15.

7. Universidade e Medicina Johns Hopkins. Centro de recursos do Coronavirus [Internet]. 2020 [citado em 4 de maio de 2020]. Disponível em: > https://coronavirus.jhu.edu/<

8. Mor G, Aldo P, Alvero AB. The unique immunological and microbial aspects of pregnancy. Nature Reviews Immunology. 2017 Aug;17(8):469.

9. Liu H, Wang LL, Zhao SJ, Kwak-Kim J, Mor G, Liao AH. Why are pregnant women susceptible to COVID-19? An immunological viewpoint. Journal of reproductive immunology. 2020 Jun 1;139:103122.

10. O'Day MP. Cardio-respiratory physiological adaptation of pregnancy. InSeminars in perinatology 1997 Aug 1 (Vol. 21, No. 4, pp. 268-275). WB Saunders.

11. Bobrowski RA. Pulmonary physiology in pregnancy. Clinical obstetrics and gynecology. 2010 Jun 1;53(2):285300.

12. Raichel L, Romanyuk V, Sergienko R, Wiznitzer A, Sheiner E. 547: Pneumonia during pregnancy: radiological characteristics, predisposing factors, and pregnancy outcomes. American Journal of 13. Obstetrics \&Gynecology. 2009 Dec 1;201(6):S203-4.

14. Meijer WJ, van Noortwijk AG, Bruinse HW, Wensing AM. Influenza virus infection in pregnancy: a review. Acta obstetricia et gynecologica Scandinavica. 2015 Aug;94(8):797-819. 